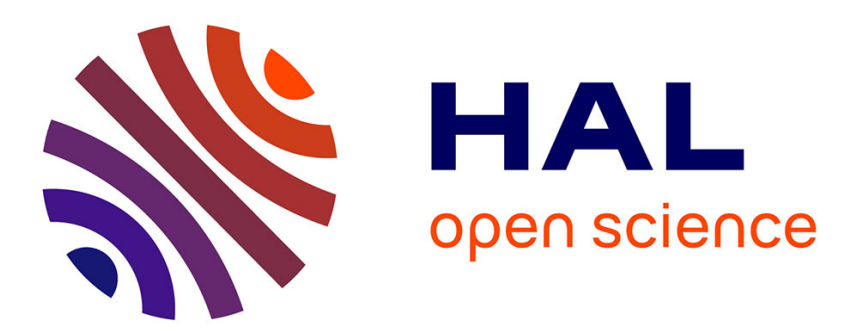

\title{
Chypre dans le nouvel ordre méditerranéen de l'époque hellénistique. Approches épigraphiques
}

\author{
Anaïs Michel
}

\section{To cite this version:}

Anaïs Michel. Chypre dans le nouvel ordre méditerranéen de l'époque hellénistique. Approches épigraphiques. Cahiers du Centre d'Etudes Chypriotes, 2016, 46, pp.289-304. hal-02505228

\section{HAL Id: hal-02505228 \\ https://hal.science/hal-02505228}

Submitted on 28 Dec 2021

HAL is a multi-disciplinary open access archive for the deposit and dissemination of scientific research documents, whether they are published or not. The documents may come from teaching and research institutions in France or abroad, or from public or private research centers.
L'archive ouverte pluridisciplinaire HAL, est destinée au dépôt et à la diffusion de documents scientifiques de niveau recherche, publiés ou non, émanant des établissements d'enseignement et de recherche français ou étrangers, des laboratoires publics ou privés. 


\title{
CENTRE D'ÉTUDES CHYPRIOTES
}

\section{CAHIER}

46, 2016

\author{
Hommage à Antoine HERMARY \\ Colloque "Chypre et les grandes îles de Méditerranée » \\ Marseille, 16-17 octobre 2015
}

Publié avec le concours de la Fondation A.G. Leventis

Édition-Diffusion De Boccard

11, rue de Médicis, F-75006 Paris 

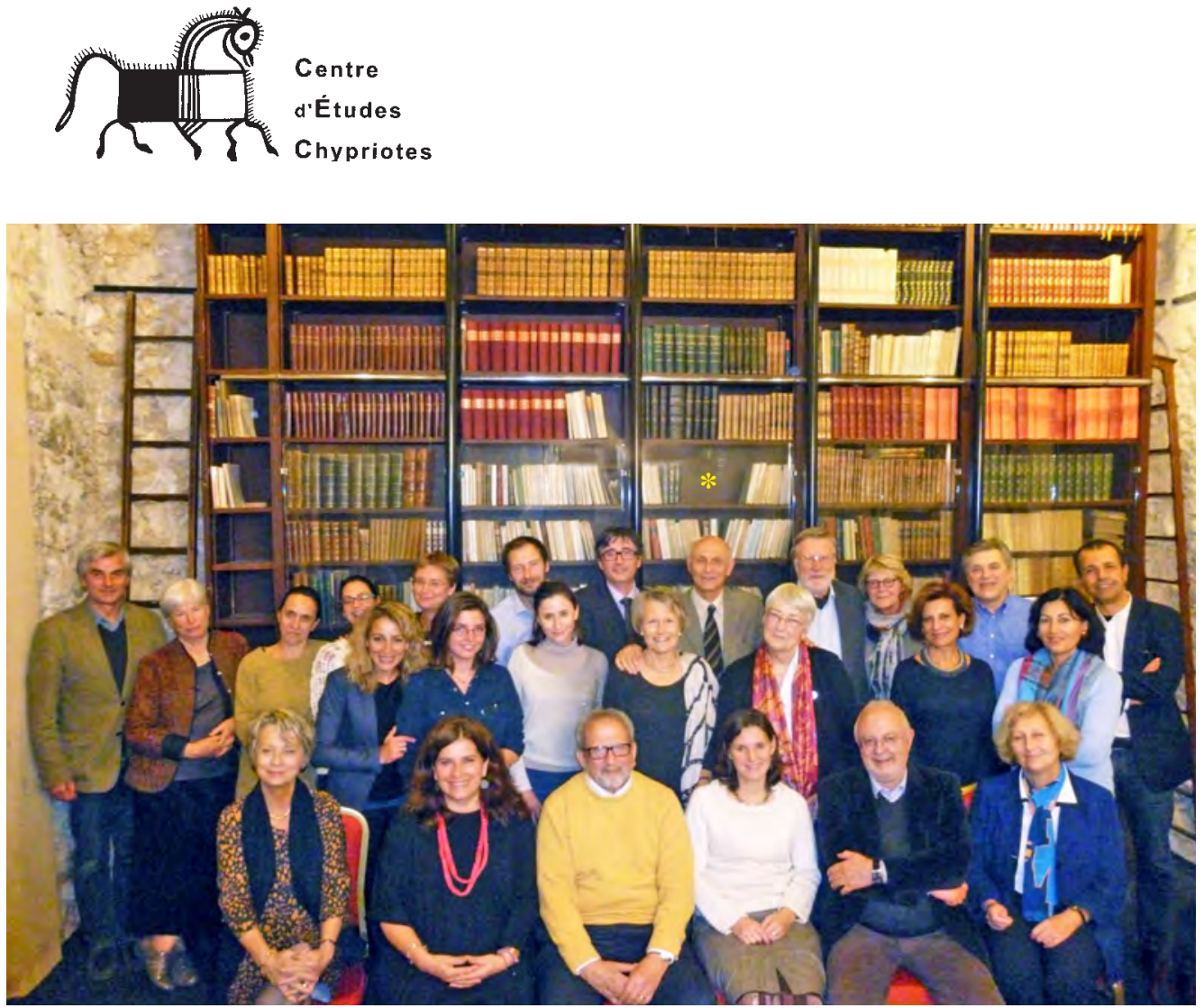

Antoine Hermary (*) et les participants au colloque, Marseille, 17 octobre 2015.

La revue Cahiers du Centre d'Études chypriotes (abrégée CCEC) publie des contributions en allemand, anglais, français, grec, italien, et rend compte d'ouvrages qui lui sont envoyés. Adresser les propositions d'articles au directeur de la revue (Centre Camille-Jullian, Aix).

Directeur de la revue: Antoine HERMARY.

Comité de rédaction : Derek COUNTS, Sabine FOURRIER, Antoine Hermary, Hartmut MATTHÄUS, Robert MERRILLEES, Marguerite YON, qui constituent aussi le Comité de lecture avec la collaboration de spécialistes extérieurs.

Maquette, mise en page : Marguerite YON.

Aix-Marseille Univ, CNRS, Minist Culture \& Com, CCJ, 5 rue du Château-de-l'Horloge, B.P. 647,

F-13094 Aix-en-Provence Cedex 2. ahermary@mmsh.univ-aix.fr

HiSoMA [Histoire et Sources des Mondes Antiques], Maison de l'Orient et de la Méditerranée Jean Pouilloux, 7 rue Raulin, F-69365 Lyon Cedex 07._sabine.fourrier@mom.fr_marguerite.yon@mom.fr 


\section{SOMMAIRE}

Avant-propos, par Antoine Hermary, Président du Centre ........................................ 7

In memoriam, Edgar J. Peltenburg, par Annie CAUBet et Gordon D. Thомas ............... 9

In memoriam, Donald M. Bailey, par Thomas KIELY .................................................. 15

\section{ACTES DU COLLOQUE DE MARSEILLE, 16-17 OCTOBRE 2015}

en hommage à Antoine Hermary

«Chypre et les grandes îles de Méditerranée

Un nouvel espace d'échanges,

de la fin du II millénaire av. J.-C. à l'époque hellénistique»

Introduction, Un parcours méditerranéen

par Sabine Fourrier, Jean-Christophe Sourisseau, Henri Tréziny

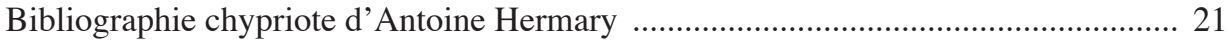

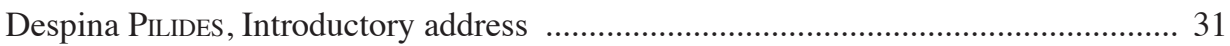

Une Méditerranée d'îles : matières premières et échanges

Marguerite Yon, Aux échelles du Levant.

Échanges commerciaux au Bronze Récent

Nota Kourou, A Cypriot Sequence in Early Iron Age Crete:

Heirlooms, Imports and Adaptations

Vasiliki Kassianidou, Copper metallurgy in Iron Age Kition

Anna Georgiadou, La diffusion de la céramique chypriote d'époque géométrique en Méditerranée orientale. Productions et réseaux de distribution

Anna Cannavò, Au fil des écritures. Plurilinguisme et plurigraphisme dans les îles méditerranéennes

Henri TRÉZINY, Des tours internes aux sanctuaires suburbains.

Regards occidentaux sur des questions chypriotes

Jean-Baptiste CAYLA, Le paysage de théonymes en Crète et à Chypre

Art et artisanat, réseaux et échos

Annie CAubet et Hélène Le MeAux, D'Alasia à Tartessos .

Ivoires et autres matières dures d'origine animale

Hartmut MatтhäUs, Kypriaka in der Siderospilia-Nekropole

von Prinias, Mittelkreta 
Lou de BARBARIN et Jean-Christophe Sourisseau, Trafics orientaux en Méditerranée occidentale. Quelques remarques sur la diffusion des styles céramiques dans le répertoire des cités grecques de Sicile orientale

Hélène Aurigny, De Chypre à Delphes : objets et réseaux en Méditerranée orientale pendant le haut-archaïsme

Reinhard SenfF, The Early Stone Sculpture of Cyprus in the Archaic Age. Questions of Meaning and External Relations

Hédi Dridi, Chypre et la Sicile : quelques réflexions sur les usages comparatifs du marbre avant le $\mathrm{III}^{\mathrm{e}}$ siècle av. J.-C.

Pauline MAILlard, Diplomates, archéologues et joggeurs aux Salines de Kition. La localisation du sanctuaire « de la Paralia»

Marcella PisANi, Modelli attici nella coroplastica siceliota di età tardo-classica. Problemi di stile, cronologia e società

\section{Un autre monde? L'époque hellénistique}

Anaïs Michel, Chypre dans le nouvel ordre méditerranéen de l'époque hellénistique. Approches épigraphiques

Demetrios Michaelides et Anne-Marie Guimier-Sorbets, Funerary architecture and other underground structures in Hellenistic Cyprus and Rhodes

Ludovic Thély, Au sujet de deux attestations de l'Aphrodite Kypris en Crète orientale à la basse époque hellénistique

\section{Conclusions}

Pierre AUPERT, Itinéraires croisés

\section{VARIA}

Robert S. MERriLleEs, Studies on the provenances of the stele of Sargon II from Larnaca (Kition) and the two so-called Dhali (Idalion) silver bowls in the Louvre

Olivier CALlOT, Les monnaies de Kition-Bamboula 387

\section{COMPTES RENDUS D'OUVRAGES}

1. I. A. TodD, The Field Survey of the Vasilikos Valley. Volume II. Artefacts Recovered by the Field Survey (SIMA 71:10), Uppsala, 2016 (S. Fourrier)

2. A. Jacobs, P. Cosyns (éd.), PoCA 8 (Posgraduate Cypriot Archaeology) Brussel 2008, Bruxelles, 2015 (R. Merrillees)

3. H. Matthäus, B. Monstadt, C. Vonhoff (éd.), PoCA 12 (Posgraduate Cypriot Archaeology) Erlangen-Nuremberg 2012, Cambridge, 2015 (A. Hermary)

4. A. Nicolaou-Konnari, C. Schabel (éd.), Lemessos: A History of Limassol in Cyprus from Antiquity to the Ottoman Conquest, Cambridge, 2015 (Ph. Trélat)

5. M. Grünbart, S. Rogge (éd.), Medieval Cyprus.

A Place of Cultural Encounter, Münster, 2015 (N. Lécuyer) 


\title{
CHYPRE DANS LE NOUVEL ORDRE MÉDITERRANÉEN DE L'ÉPOQUE HELLÉNISTIQUE Approches épigraphiques
}

\section{Anaïs MICHEL}

\begin{abstract}
In this paper I aim to shed light on one of today's most dynamic fields of Cypriot studies, i.e. the Hellenistic period and the Ptolemaic occupation of the island from 295/4 to 31 B.C. Based on a PhD project devoted to the reading of the Greek inscriptions of Hellenistic Cyprus and the study of the Cypriot society under Ptolemaic rule, it outlines the specificities of the Hellenistic period in Cyprus, and discusses its traditional representation as a «brand new world ». It will first recall Cyprus' political, strategic and economical importance in the Ptolemaic foreign policy, and then focus on the installation of an administrative and military staff on the island. These synthetic lines will highlight the new Mediterranean networks of the Ptolemaic period. In the last part of the article I will focus on the role taken by the Cypriote elite both in local and external contexts.
\end{abstract}

L'un des objectifs de cette rencontre était de montrer qu'une île, malgré des particularités qui peuvent, dans le cas de Chypre, confiner au domaine de la légende, n'est pas un monde clos, un univers isolé en Méditerranée. L'examen de plusieurs phénomènes (touchant notamment aux pratiques funéraires, artistiques, linguistiques et commerciales) a montré que les îles se rencontrent et s'intègrent au contraire dans une pluralité de réseaux, et ce dès la fin du $2^{\mathrm{e}}$ millénaire av. n. è. Identifier et explorer ces réseaux requiert d'adopter une double démarche : l'étude fine d'un phénomène local ou d'un matériel ciblé, enrichie d'un goût certain pour l'aventure et le voyage, permettant de «naviguer » entre les différentes échelles et les différents niveaux de référence déployés sur les scènes, plurielles, de l'archéologie et de l'histoire méditerranéennes.

Dans cet ensemble varié et néanmoins lié, j'ai choisi de focaliser mon attention sur Chypre, en sorte que l'objet de l'étude présentée ici rejoint en partie celui de mes recherches doctorales ${ }^{1}$ consacrées à la société chypriote hellénistique. Si cette étude porte exclusivement sur Chypre, il semble néanmoins impossible d'examiner la situation de l'île à l'époque hellénistique sans tenir compte des spécificités inhérentes à cette période, dont l'un des traits caractéristiques tient justement dans l'enchevêtrement des histoires

1. Dans le cadre d'une thèse menée depuis septembre 2013 sous la double direction du Professeur Michaelides (University of Cyprus) et du Professeur Hermary (AMU - Centre Camille Jullian). 
locales au sein d'un tissu universel - dans les limites du sens que l'on peut donner à ce terme dans l'Antiquité méditerranéenne ${ }^{2}$.

Ainsi, l'histoire de Chypre entre 323 et $30^{3}$ est marquée très fortement d'une part par son intégration dans l'empire ptolémaïque, et d'autre part par son insertion dans des réseaux méditerranéens renouvelés. Du fait de sa situation particulière dans l'histoire du monde hellénistique, Chypre représente un terrain de recherche épigraphique riche et, par certains aspects, unique, nous permettant de saisir les contours et parfois, les caractères et les nuances d'une société complexe engagée pour un temps remarquablement long dans un contexte politique relativement stable ${ }^{4}$.

La question qui constitue le point de départ de cette enquête porte donc sur la situation de l'île dans les réseaux méditerranéens de l'époque hellénistique. Deux grands axes d'étude s'imposent : d'abord la position de Chypre vis-à-vis d'Alexandrie et son intégration dans l'empire lagide ; puis, au vu des grandes tendances du corpus, l'exploration des réseaux de contacts dans lesquels elle s'insère. Ce second aspect, que je traiterai en deux temps, permettra de considérer d'une part la présence de nombreux étrangers à Chypre, et d'autre part celle des Chypriotes hors de leur île. Je m'appuierai sur quelques exemples précis pour traiter ces deux derniers ensembles.

\section{L'intégration dans l'empire lagide}

L'époque hellénistique représente une nouvelle donne géopolitique, effective à l'échelle du bassin méditerranéen, qui se traduit à Chypre par la disparition du système politique préexistant. Les conflits qui voient le jour entre les Diadoques pour l'annexion de l'île puis son rattachement, en deux temps ${ }^{5}$, au royaume lagide entraînent ainsi la mort des cités-royaumes de l'époque archaïque et classique. À l'orée de l'époque hellénistique, l'île de Chypre présente des intérêts stratégiques déterminants du point de vue des monarchies naissantes et la bataille pour son contrôle revêt des aspects militaires, politiques et économiques. Pour les Ptolémées, la possession de Chypre permet la sécurisation des frontières de l'Égypte et la protection du Delta, tout en leur assurant une position stratégique en face de la Syrie séleucide. À côté de cet aspect militaire, l'exploitation des ressources naturelles chypriotes ${ }^{6}$ est un autre moteur important de la

2. Sur l'histoire du monde hellénistique, voir notamment Cl. Préaux, Le monde hellénistique, 2 vol., Paris, 1978 ; Will 2003 ; C. Grandjean, G. Hoffmann, J.-Y. Carrez-Maratray, Le monde hellénistique, Paris, 2008.

3. Ces dates, qui correspondent aux dates de mort d'Alexandre le Grand et de Cléopâtre VII, la dernière représentante de la dynastie lagide, coïncident avec les limites chronologiques de la période dite hellénistique. La chronologie chypriote épouse parfaitement ces limites.

4. La domination lagide sur Chypre ne connaît pas de solution de continuité pendant près de deux-cent cinquante ans, si ce n'est en 168, au moment de l'incursion séleucide d'Antiochos IV (Will 2003, vol. 2, p. 322). Le rattachement provisoire de l'île à la province romaine de Cilicie de 58 à 48 amorce quant à lui la fin de la période lagide à Chypre (ibid., p. 522-525).

5. En 312, puis (à la suite de la défaite de Ptolémée contre Antigone et Démétrios à Salamine en 306) en 295/4 ; c'est cette dernière date qui inaugure traditionnellement l'ère lagide à Chypre.

6. On pense bien-sûr au bois, nécessaire à la construction et à l'entretien d'une flotte, et au cuivre, 
conquête lagide. Bien-sûr, ce serait sans doute une erreur de chercher à isoler l'histoire hellénistique de Chypre des périodes qui l'ont précédée. Si la disparition tragique des derniers représentants des cités-royaumes est un événement capital qui correspond à un réel tournant dans l'histoire de Chypre, il est certainement précipité de penser que la conquête lagide transforme radicalement la société chypriote ${ }^{7}$. Les perspectives actuelles de la recherche s'orientent ainsi désormais vers la notion de transition ${ }^{8}$.

L'occupation définitive de Chypre par Ptolémée dès 295/4 entraîne néanmoins des conséquences importantes sur la structure politique et sociale de l'île. Cette dernière se voit, pour la première fois, unifiée sous la tutelle d'une puissance extérieure ${ }^{9}$. L'intégration dans l'empire lagide se traduit par une véritable mainmise des agents ptolémaïques dans tous les domaines-clés de l'administration et de l'économie chypriotes et contribue à souligner la singularité de la relation de Chypre avec les Ptolémées. La Nouvelle Paphos devient le centre administratif de l'île, en même temps que le siège d'une cour d'Alexandrins en fonction à Chypre et réunis autour de la personnalité du stratège. Principal représentant du pouvoir lagide à Chypre et issu du tout premier cercle de la cour alexandrine, celui-ci est investi du pouvoir à la fois militaire et administratif. À lui seul, il incarne la présence du monarque dans l'île, et l'évolution de son champ d'action au cours de la période est significative de la politique lagide mise en place hors

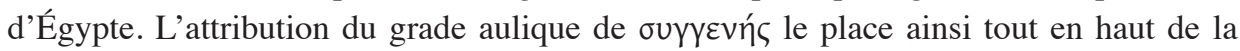
hiérarchie de cour lagide, même - ce qui est remarquable - au-dessus de l'épistratège de la chôra égyptienne ou encore du diœè̀te, le ministre des finances du royaume ${ }^{10}$. Cette

mais aussi à l'exploitation des savoir-faire locaux, dans le domaine de la construction navale ou de la production monétaire par exemple. Sur ce dernier point, on sait que les ateliers de Salamine, de Kition et de Paphos sont responsables d'un assez gros volume d'émissions sous les Ptolémées ; un quatrième atelier a ponctuellement été mis en service à Amathonte (J. Olivier, H. Aumaître, « L'atelier monétaire d'Amathonte et la Sixième Guerre de Syrie (170/9-169/8) », Bulletin de la Société française de Numismatique, 2014, p. 26-36).

7.A.-M. Collombier ( «La fin des royaumes chypriotes : ruptures et continuités », Transeuphratène 6, 1993, p. 119-147) est la première à poser la question des « continuités » entre l'époque des royaumes et l'ère lagide à Chypre.

8. C'est autour de la notion de transition que s'est tenu le colloque de Nicosie consacré en 2002 à cette période charnière de l'histoire antique de Chypre (P. Flourentzos [éd.], From Evagoras I to the Ptolemies. The transition from the Classical to the Hellenistic Period in Cyprus. Proceedings of the International Archaeological Conference, Nicosia 29-30 November 2002, Nicosie, 2007). Cette même notion est mise en exergue par S. Lejeune (Chypre en transition. Les cités chypriotes de la fin des Royaumes autonomes à la mise en ordre lagide, IV -III siècles av. J.-C., thèse inédite, Université de Paris Ouest-Nanterre, 2013). Voir également M. Hatzopoulos, «Chypre, de la multiplicité des royaumes à l'unité de la province lagide », CCEC 39, 2009, p. 228 ; Papantoniou 2012.

9. Voir A. Mehl, « Cypriot City Kingdoms: No Problem in the Neo-Assyrian, Late Egyptian

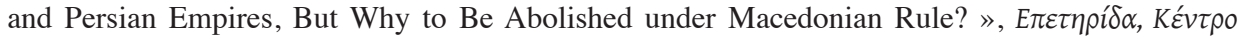

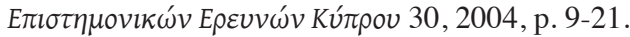

10. Il est généralement accepté que le poste de stratège de Chypre a représenté l'un des degrés les plus élevés dans la hiérarchie aulique lagide. Voir Bagnall 1976, p. 38-49 ; Mooren 1977, p. 181. 
distinction tend à souligner l'importance particulière qui revient à Chypre dans l'empire lagide ${ }^{11}$.

Une analyse des diverses fonctions assignées au personnel de la cour lagide installé à Chypre permet de constater que l'autorité royale s'y exprime dans des domaines aussi variés que ceux du commandement militaire, du culte ou de l'industrie ${ }^{12}$. D'autre part, le pouvoir lagide s'impose physiquement dans le paysage hellénistique de Chypre avec l'installation de garnisons. Leur organisation n'est pas clairement documentée par les données archéologiques ${ }^{13}$; mais la mention dans les inscriptions de koina militaires, de mercenaires et d'officiers, offre l'indice sûr de l'existence d'un système militaire hiérarchisé et d'une visibilité forte des troupes de soldats à Chypre ${ }^{14}$.

Le domaine religieux représente un autre aspect de l'intégration de Chypre dans l'empire lagide, particulièrement bien documenté par les sources épigraphiques. À partir de Polykratès d'Argos (203-197), le stratège de l'île se voit attribuer le titre d'ó $\rho x เ \varepsilon \rho \varepsilon u ́ \varsigma$ (grand-prêtre) qui place sous son autorité la totalité des sanctuaires chypriotes ${ }^{15}$. On sait par ailleurs que, dans le domaine religieux, la législation alexandrine est parfois appliquée à Chypre : c'est le cas notamment pour le versement de l'apomoira ${ }^{16}$, une

L'importance de Chypre est par ailleurs soulignée par les titres auliques des gouverneurs des cités : si à Chypre ils portent régulièrement le titre d'archisômatophylaques, les deux gouverneurs de la ville de Théra entre 170 et 164 puis entre 163/2 et environ 145 appartiennent, eux, à la classe inférieure des diadoques. Ces remarques ainsi que le schéma hiérarchique qu'on adopte ici sont issus des travaux de Mooren. Voir notamment Mooren 1977, p. 196.

11. Mooren 1977, p. 208-209 : «Le fait que le stratège de Chypre se trouve au sommet de la hiérarchie indique que le souci majeur des Lagides concerne cette perle de leurs possessions extérieures ». Voir désormais Fischer-Bovet 2014, p. 157.

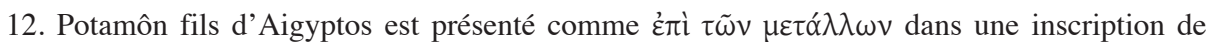
Paphos : Mitford 1961, p. 39, nº 107 ; Bagnall 1976, p. 74 ; Cayla 2006 ; Mehl 2016, p. 251-253.

13. Voir néanmoins les récentes avancées issues des fouilles dans la région de Paphos: C. Balandier, «Des anciennes capitales de royaumes aux nouvelles villes portuaires : réflexions sur l'évolution du réseau urbain de Chypre à l'époque hellénistique », dans C. Balandier, Chr. Chandezon (éd.), Institutions, sociétés et cultes de la Mediterranée antique (Mélanges Claude Vial), Scripta Antiqua 57, 2014, p. 179-209 ; D. Vitas, «The Foundation of Nea Paphos: A new Cypriot City or or a Ptolemaic katoikia ? », dans Balandier 2016, p. 241-248.

14. Concernantl'aspect militaire del'occupation lagide, le point est fait par A. Mehl, «Militärwesen und Verwaltung der Ptolemäer in Zypern », Rivista di cultura classica e medioevale 38, 1996, p. 215-260. Pour une lecture globale de la domination lagide à Chypre, voir Mitford 1953.

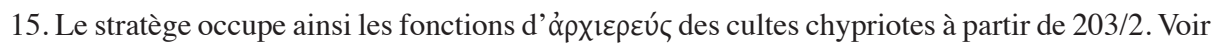
à ce sujet Bagnall 1976, p. 73.

16. Voir Bagnall 1976, p. 228 ; W. Clarysse, K. Vandorpe, « The Ptolemaic Apomoira », dans $\mathrm{H}$. Melaerts (éd.), Le culte du souverain dans l'Égypte ptolémä̈que au III siècle avant notre ère. Actes du colloque international, Bruxelles 10 mai 1995 (Studia Hellenistica 34), 1998, Louvain, p. 5-42. Sur la réforme de la taxe de l'apomoira en Égypte : S.G. Caneva, «Queens and Ruler Cult in Early Hellenism: Observations on festivals, and on the administration and ideological meaning of cults », Kernos 25, 2012, p. 88. S. Lejeune revient sur un éventuel rapprochement du corpus 
taxe en vigueur à Alexandrie et dont on retrouve la trace dans la cité d'Arsinoé (ancienne Marion) ${ }^{17}$. Sachant que les revenus produits par cette taxe agricole sont reversés au culte d'Arsinoé II Philadelphe, force est de constater l'opportunité d'une telle mesure à Chypre où le culte de la Déesse Philadelphe se diffuse et s'exprime de façon notable ${ }^{18}$. Un personnel religieux réservé à son culte lui est en outre assigné dans l'île ${ }^{19}$.

L'intégration, à la fois en matière de durée et de degré d'intensité, de la province chypriote à l'empire ptolémaïque est remarquable. Les inflexions de la politique lagide à Chypre permettent de déterminer plusieurs séquences distinctes dans l'histoire hellénistique de l'île. Ainsi, plusieurs facteurs entraînent une évolution de la relation de l'île avec l'Égypte lagide autour du milieu du II ${ }^{\mathrm{e}} \mathrm{s}$. Les incursions d'Antiochos IV à Chypre - et jusqu'aux portes d'Alexandrie - au cours de la Sixième Guerre de Syrie ${ }^{20}$ provoquent de fait un resserrement de la stratégie défensive lagide sur Chypre, ce qui se traduit, du point de vue de l'île, par une intégration plus étroite au royaume. En 142, les troupes lagides se retirent de leurs dernières bases en mer Égée, et le repli sur Chypre entraîne l'attribution du titre de navarque au stratège de l'île ${ }^{21}$. À partir de cette date, Chypre devient le poste avancé principal des Ptolémées hors d'Égypte, caractère qui ne fait que s'accentuer avec la perte des dernières possessions lagides au cours des II $^{\mathrm{e}}$ et $\mathrm{I}^{\mathrm{er}}$ siècles.

Lorsque le poste de strategos revient finalement à un Ptolémée, au cœur des secousses dynastiques ${ }^{22}$ qui peu à peu défont la puissance lagide dès la deuxième moitié du $\mathrm{II}^{\mathrm{e}} \mathrm{s}$., des liens plus personnels semblent se créer entre Chypre et les Ptolémées. On assiste alors à l'ascension de certains Chypriotes au sein de la hiérarchie aulique lagide ; c'est la première fois dans l'épigraphie chypriote que l'on voit s'illustrer des individus d'origine locale à des postes capitaux - tel que celui d'antistrategos - et jouir du statut de бuүүદvท́ jusque là réservé aux dignitaires de la cour alexandrine ${ }^{23}$.

des offrandes de Kafizin avec la taxe de l'apomoira : Lejeune 2014, p. 298-300 et n. 170 pour la bibliographie antérieure. Pour les pratiques religieuses liées au culte d'Arsinoé II, voir la synthèse récente de S. Caneva, « Ruler Cults in practice: Sacrifices and libations for Arsinoe Philadelphos, from Alexandria and Beyond », dans T. Gnoli, F. Muccioli (éd.), Divinizzazione, culto del sovrano e apoteosi Tra Antichità e Medioevo, Bologne, 2014, p. 85-116.

17. LBW, n 2783. Voir également Anastassiades 1998, p. 138, nº 3.

18. Sur ce point, voir la documentation réunie dans Anastassiades 1998.

19. On connaît une canéphore d'Arsinoé à Idalion ( $\left.K B \mathrm{~V}, \mathrm{n}^{\circ} 82\right)$. Un tambour de colonne de provenance inconnue mentionne également un prêtre d'Arsinoé (Anastassiades 1998, p. 140, n² 28).

20. Voir supra, n. 5.

21. Séleukos fils de Bithys est le premier à porter ce titre à Chypre. Voir Bagnall 1976, p. 47.

22. On pense notamment aux troubles opposant Ptolémée Philométor à son frère Évergète II ou encore, à la génération suivante, Ptolémée Sôter II à son frère Alexandre I. Voir Will 2003, vol. 2, p. $361-365 ; 429-432 ; 443-445$.

23. Antistrategos: Potamôn fils d'Aigyptos : Mitford 1961, p. 39, n 107 ; Cayla 2006 et Mehl 2016, p. 251-253; pour l'attribution de ce titre exceptionnel à Chypre, ainsi que celui d'éđì $\tau \tilde{\omega} v$ 
Si l'on ${ }^{24}$ a parfois brossé le tableau d'une société étouffée par l'occupation lagide, les sources épigraphiques montrent que les institutions civiques ne restent pas sans voix à l'époque hellénistique. Il semblerait même que les Ptolémées aient, d'une certaine façon, encouragé leur diffusion, malgré les limites nécessairement posées aux prérogatives des cités par l'occupation militaire de l'île et son intégration au sein d'un empire. D'après Bagnall $^{25}$, treize villes chypriotes bénéficient du statut civique à l'époque hellénistique. On trouve dans les sources épigraphiques certains indices de cette activité ${ }^{26}$. Alors que la cité n'appartient pas au peuple aux époques antérieures ${ }^{27}$, on voit apparaître dans les inscriptions la formule $\grave{\eta} \pi o ́ \lambda ı \varsigma ~ \tau \tilde{\omega} v$ suivie du génitif du nom des habitants de la cité.

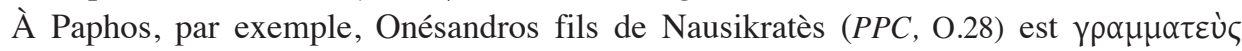

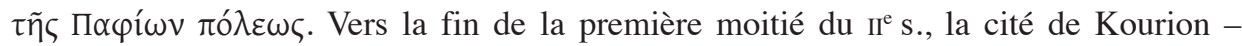

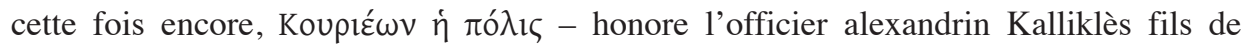
Kalliklès ${ }^{28}$. D'autre part, des organes civiques commencent à apparaître ${ }^{29}$ dans les textes

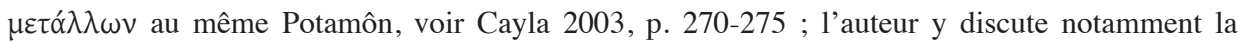
datation de cette inscription, ce qui l'amène à revoir la chronologie de ces institutions à Chypre.

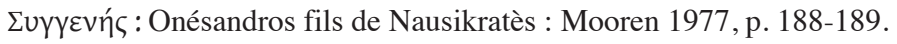

24. On doit cette vision de la présence lagide à Chypre à M.I. Rostovtzeff (Social and Economic History of the Hellenistic World, Oxford, 1953 [1941], p. 339, cité par Bagnall 1976, p. 57-58). Si l'historiographie tend à s'écarter de cette lecture réductrice de l'histoire hellénistique de Chypre, notamment depuis T.B. Mitford (« The Status of Cypriot Epigraphy: Cypriot Writing, Minoan to Byzantine » Archaeology V-3, 1952, p. 155 ; Id. 1953) et R.S. Bagnall (voir, par exemple, ses remarques conclusives sur les cités chypriotes à l'époque hellénistique : Bagnall 1976, p. 67-73), celle-ci transparaît encore dans l'œuvre d'éminents spécialistes de l'épigraphie chypriote : voir notamment I. Michaelidou-Nicolaou, dans la préface de PPC (on lit notamment p. 24, «Under the Ptolemies Cyprus had no political life of its own. It was the first but also the last Ptolemaic possession, whose rich mines, forests and geographical position offered opportunities to the Ptolemies for exploitation. What else Cyprus has to show for this period comes within the general picture of the Hellenistic koine, which was common to the whole Hellenistic world.»), ou encore les remarques de P. Roesch dans son étude des inscriptions hellénistiques de Salamine : «Les Lagides à Salamine : épigraphie et administration », dans Salamine de Chypre, Histoire et Archéologie, État des recherches, Paris, 1980, notamment p. 256.

25. Bagnall 1976, p. 66-67.

26. Voir notamment Młynarczyk 1990, p. 136-137.

27. A. Cannavò, Les royaumes chypriotes archaïques : études des sources textuelles, thèse inédite, Université Lumière Lyon 2-Scuola Normale Superiore di Pisa, 2011, p. 183 et n. 97.

28. L'inscription est gravée sur une base découverte en 1998 sur l'acropole : PPC, K.6 ; I. Nicolaou, "The city of Kourion honours Kallikles, son of Kallikles of Alexandria », Acta XII congressus internationalis epigraphiae graecae et latinae : provinciae imperii romani inscriptionibus descriptae, Barcelona, 3-8 Septembris 2002, Barcelone, 2007, p. 1013-1016 et

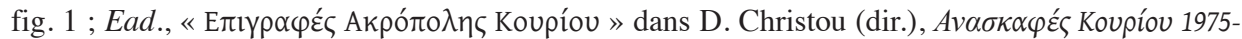
1998, vol. II, Nicosie, 2013, p. 315, nº 1.

29. Il faut insister sur le fait que cette « innovation » est avant tout épigraphique, et qu'en aucun cas elle n'autorise à tirer des conclusions quant à l'absence d'institutions civiques aux époques antérieures, ni sur la prétendue « hellénisation» de l'île par les Lagides. Voir le chapitre consacré 
épigraphiques : par exemple, le décret I. Kourion $32^{30}$ en l'honneur d'un Sidonien porte la mention de la boulè et des archontes de la cité de Kourion.

Une autre fonction locale est bien documentée à Chypre : celle du politikos strategos ${ }^{31}$. À Kition par exemple, où le magistrat Apollodôros ${ }^{32}$ fills de Dionysios, strategos politikos, est honoré par la cité qui lui décerne une statue. Le statut et les fonctions attachés à ce stratège local font débat ${ }^{33}$.

Enfin, le gymnase est une institution particulièrement dynamique à l'époque hellénistique. Le gymnasiarque, qui est vraisemblablement un élu d'origine locale, possède une autorité effective et intervient de façon systématique dans le dialogue entre les cités et le pouvoir royal. Ainsi, Onésikratès fils d'Onésikratès ( $P P C, \mathrm{O} .33)$, gymnasiarque à Amathonte dans les années 165-145, est l'auteur d'une dédicace monumentale en l'honneur de Ptolémée Philométor, son épouse et ses enfants ${ }^{34}$.

Les sources épigraphiques tendent par ailleurs à montrer que la communication entre les rois et la population de l'île n'est pas unilatérale. Un document exceptionnel provenant de Palaepaphos en est une remarquable illustration ; Ptolémée Philadelphe y honore d'une statue Pyrgotélès fils de Zoès ${ }^{35}$, l'architecte naval en charge de la construction de deux bâtiments de vingt et trente rangs de rames : c'est probablement un homme d'origine locale comme l'attestent l'onomastique et l'absence d'ethnique dans la dédicace. Le savoir-faire des artisans chypriotes continue donc d'être reconnu à l'époque

à cette question par G. Papantoniou (2012, p. 28-52). J.-B. Cayla exprime des réserves quant à l'apparition des institutions civiques à Chypre avant la fin de la période hellénistique ("Bulletin épigraphique », REG 118, 2005, p. 575 et, pour Paphos, Cayla 2003, p. 280).

30. Le décret est gravé sur une stèle rectangulaire en calcaire gris, moulurée, dont quatre fragments subsistent. R.S. Bagnall, Th. Drew-Bear, « Notes on the history of Kourion », Chronique d'Égypte 49, 1974, p. 179-183.

31. Voir notamment Robert 1965, p. 181-182, n 453 ; Mitford 1961, p. 21 ; Mooren 1977, p. 196 ; Launey 1987, p. 1016 ; Młynarczyk 1990, p. 135.

32. $P P C, \mathrm{~A} .62 ; K B \mathrm{~V}, \mathrm{n}^{\circ} 2025$.

33. L'origine, locale ou non, de ces magistrats est discutée. Pour L. Mooren (1977, p. 196) et J. et L. Robert (1965, p. 181-182, à propos du n 453), il s'agit vraisemblablement de Chypriotes. I. Michaelidou-Nicolaou, quant à elle, fait figurer ces magistrats parmi les «other Ptolemaic officials» dans son index à $P P C$. D'autres fonctions relèvent à Chypre de la magistrature locale. On pense par exemple à celle d'hégétor, attestée à Amathonte (Hermary 1999, p. 51-54) et à Paphos (Cayla 2003, p. 276-277). Le grammateus Kallippos (Mitford 1961, p. 36, nº 98), secrétaire (ici de la boulè et du démos, puis de la polis de Paphos), entre également dans cette catégorie, tout comme les agoranomes, magistrats mentionnés dans une inscription de Salamine ( $\mathrm{Sal}$. XIII, n 36) et dont l'un des représentants apparaît sur un poids de plomb $(P P C, \mathrm{Z} .4)$.

34. Voir récemment $\mathrm{P}$. Aupert, « Amathonte hellénistique et impériale : l'apport des travaux récents », CCEC 39, 2009, p. 31-32.

35. РРС, П.74 et Z.15 ; M.-Chr. Hellmann, Choix d'inscriptions architecturales traduites et commentées, Lyon, 1999, p. 98-99, n 40 ; Cayla 2003, p. 226, n 61 (et dans ce volume, où l'auteur avance une nouvelle hypothèse de datation). 
hellénistique. Réciproquement, les dédicaces de Chypriotes en l'honneur des Ptolémées ou de leurs représentants dans l'île fleurissent : on observe ainsi une concentration remarquable d'hommages destinés directement aux rois ou aux dignitaires lagides dans le sanctuaire d'Aphrodite à Palaepaphos, véritable vitrine du pouvoir ptolémaïque à Chypre. L'Aphrodite grecque, « adoptée » depuis longtemps déjà par la Grande Déesse en son sanctuaire, contribue par son rayonnement international à asseoir le prestige des Lagides. Dans cette relation, où l'on emploie avec aisance le langage des honneurs propre à l'époque hellénistique ${ }^{36}$, les Chypriotes accueillent apparemment avec chaleur - et un sens du politique éprouvé - le culte des rois et des reines lagides : Arsinoé II est de loin la mieux représentée ${ }^{37}$ car son culte semble véritablement s'être fondu dans le paysage religieux chypriote, mais des pratiques cultuelles attestées par l'épigraphie s'attachent également à d'autres membres de la dynastie ${ }^{38}$.

Si l'on reprend brièvement les principaux points de ce premier développement, il faut insister sur le processus d'intégration de l'île de Chypre au sein du royaume et de l'administration ptolémaïques qui contribue, par sa longévité et par son intensité, à lui conférer un statut extraordinaire - comparable, en un sens, à celui de l'Égypte. Le corpus épigraphique de Chypre reflète cette configuration. Souverains et dignitaires lagides y sont omniprésents, intervenant dans tous les domaines de la vie politique, militaire, économique et religieuse. Néanmoins, ce premier constat ne permet pas de conclure que l'occupation alexandrine étouffe la société chypriote : une relation active et bilatérale se met en place, au sein de laquelle les Chypriotes et leurs cités trouvent des voies d'expression.

36. Ainsi, comme dans le reste du monde hellénistique, ce sont les notions d'arétè, d'euergésia et d'eunoia qui composent les thèmes principaux du corpus des inscriptions honorifiques de Chypre.

37. Voir à ce sujet Anastassiades 1998.

38. On sait, par exemple, par une inscription de Chytroi (T.B. Mitford, « Further contributions to the epigraphy of Cyprus » AJA 65, 1961, p. 129-131, n²9c) qu'y était célébré l'anniversaire de Ptolémée Philométor. Une onochoè provenant de Kourion (mais probablement importée d'Égypte), et portant le nom de Ptolémée IV (peut-être un élément appartenant à un ensemble de vaisselle rituelle), a été étudiée par Mitford (I.Kourion 75). D'autres textes font état de prêtres du culte royal : ainsi, le stratège Hélénos est le prêtre à vie d'une reine Cléopâtre (Sal. XIII, ${ }^{\circ}{ }^{81}$ ). Par ailleurs, plusieurs inscriptions font mention de la fondation de sanctuaires du culte royal lagide (Ptolémaia) : par exemple, celui d'Onèsandros à Paphos (Mitford 1961, p. 40, n 110). Enfin, l'activité, importante, des technites dionysiaques à Chypre n'est sans doute pas sans rapport avec le culte royal, voir S. Aneziri, «Zwischen Musen und Hof: Die dionysischen Techniten auf Zypern », ZPE 104, 1994, p. 179-198; A. Anastassiades, « Behind masks: The artists of Dionysos in Ptolemaic Cyprus », RDAC 2009, p. 195-204 (plus généralement, se référer à Fr. Dunand, « Les associations dionysiaques au service du pouvoir lagide (III ${ }^{\mathrm{e}} \mathrm{s}$. av. J.-C.) », L'association dionysiaque dans les sociétés anciennes. Actes de la table ronde (Rome, 24-25 mai 1984), Rome, 1986, p. 85-104, et plus récemment Br. Le Guen, Les Associations de Technites dionysiaques à l'époque hellénistique, Nancy, 2001). J.-B. Cayla, «Antoine, Cléopâtre et les technites dionysiaques à Chypre », $B C H$ à paraitre. Une mention des basilistes : voir PPC, N.21. 


\section{Chypre : une zone d'intenses contacts en Méditerranée}

Le catalogue prosopographique constitué par Inô Nicolaou en 1976 - Prosopography of Ptolemaic Cyprus ${ }^{39}$ - représente un instrument de travail indispensable pour l'étude de la société chypriote à l'époque hellénistique. L'un des traits caractéristiques qui se dégage de la somme des documents réunis pour la période de la domination lagide réside certainement dans la part importante prise par les étrangers dans le corpus. Presque 70 ethniques extérieurs à l'île de Chypre sont recensés dans le corpus des inscriptions hellénistiques, pour un total de 125 entrées correspondant à des individus porteurs d'ethniques étrangers. Parmi ces ethniques règne la plus grande diversité, les textes attestant la présence de Grecs - continentaux et insulaires - de Macédoniens, Thraces, Épirotes, Illyriens, et de Micrasiatiques, mais aussi de Phéniciens, Perses, Babyloniens, Égyptiens et Cyrénéens ${ }^{40}$. La situation de Chypre dans l'histoire hellénistique justifie cet afflux massif d'étrangers et nous permet d'envisager deux grands groupes principaux : les dignitaires de la cour lagide occupant des fonctions administratives ou militaires dans l'île, et les mercenaires stationnés dans les différentes garnisons.

Parmi les dignitaires issus des premiers cercles alexandrins, la diversité des ethniques contribue à esquisser le portrait d'une aristocratie venant de différentes régions de l'espace hellénistique ; les Grecs, bien-sûr, y sont omniprésents. À partir des données épigraphiques ${ }^{41}$, on peut ainsi identifier :

- parmi les stratèges de l'île (ou navarques avant 142) : Kallikratès ( $P P C, \mathrm{~K} .9)$ de Samos ; Pélops ( $P P C, \Pi .18)$ de Macédoine ; Polykratès $(P P C, \Pi .34)$ d'Argos ; un stratège anonyme de Lamia ( $P P C, A .99)$; Séleukos $(P P C, \Sigma .5)$ de Rhodes qui est aussi Alexandrin $^{42}$; Hélénos ( $P P C$, E.5) de Cyrène ;

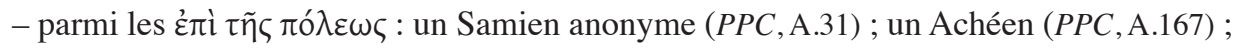
Aristos (PPC, A.127) de Chios ; Agias (PPC, A.11) de Crète ; Mélankomas (PPC, M.10) d'Étolie ;

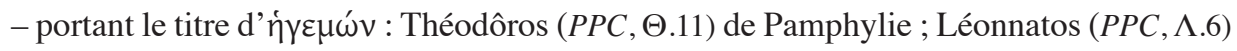
de Chios ; Praxagoras $(P P C, \Pi .45)$ de Crète ; Sôphanès $(P P C, \Sigma .53)$ d'Achaïe ;

39. Abrégé $P P C$. Sans être complet, le catalogue représente un point de départ incontournable pour la recherche épigraphique de la période hellénistique à Chypre. Le volume compte en tout 866 entrées classées par ordre alphabétique.

40. Michaelidou-Nicolaou 1967, 1968 et 1969.

41. Nous ne mentionnons que les personnages dont l'origine est assurée et la fonction à Chypre connue ; ils figurent une seule fois dans la liste, par ordre alphabétique grec et au poste le plus élevé qu'ils aient occupé au cours de leur carrière à Chypre (telle que nous pouvons la reconstituer partiellement à partir des inscriptions).

42. Les ethniques peuvent avoir une valeur honorifique. C'est peut-être également le cas pour le navarque Kallikratès de Samos, fameux promoteur du culte d'Arsinoé II (H. Hauben, Callicrates of Samos. Contribution to the Study of the Ptolemaic Admiralty [Studia Hellenistica 18], Louvain, 1970 ; Id., « Cyprus and the Ptolemaic navy », RDAC 1987, p. 213-226), qui pourrait être originaire de Paphos, voir Cayla 2003, $\mathrm{n}^{\text {os }} 62$ et 67, et p. 228 (avec bibliographie). 


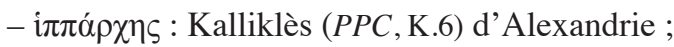

- $\gamma \rho \alpha \mu \mu \alpha \tau \varepsilon u ́ \varsigma$ des troupes : un Crétois de Hiérapytna (PPC, I.3).

Les personnages cités ci-dessus se répartissent dans les différents niveaux de la hiérarchie aulique, telle qu'elle a été fixée par L. Mooren ${ }^{43}$ : (dans l'ordre décroissant)

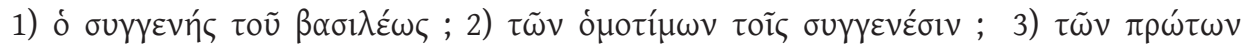

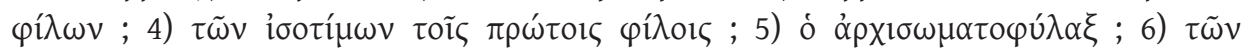

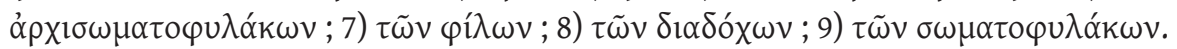

Les mercenaires étrangers sont nombreux dans le corpus des inscriptions hellénistiques ${ }^{44}$. Ces soldats nous sont surtout connus par l'épigraphie funéraire ${ }^{45}$. La nature de ces textes varie de façon conséquente, allant de la simple notation du nom et du patronyme auxquels s'ajoute l'ethnique du défunt, aux épigrammes funéraires et stèles peintes ${ }^{46}$ représentant le guerrier en armes.

Des soldats au service des Ptolémées sont connus à Chypre dès le début de la période, même s'il faut attendre la deuxième moitié du II $^{\mathrm{e}} \mathrm{s}$., et le règne de Ptolémée VIII Évergète II, pour qu'apparaisse explicitement un regroupement des mercenaires par koina. Citons, à titre d'exemple, le Crétois Praxagoras ${ }^{47}$, mort à Kition, et dont l'épigramme funéraire, composée de deux distiques élégiaques, est inscrite sur une plaque de marbre de Paros, aujourd'hui conservée au British Museum. Cette inscription représente l'unique épitaphe de chef militaire connue à Chypre et doit être datée du début du $\mathrm{II}^{\mathrm{e}} \mathrm{s}$. La fonction militaire de Nikogénès de Kalymnos ${ }^{48}$, mort à Amathonte au III $^{\mathrm{e}} \mathrm{s}$. n'est pas spécifiée dans son épigramme funéraire gravée sur une stèle peinte, également conservée au British Museum ${ }^{49}$. Pourtant ce texte, accompagné d'une figure peinte représentant un guerrier en armes, nous oriente une fois encore vers le groupe des mercenaires lagides.

Le système de recrutement des armées hellénistiques ${ }^{50}$ est étroitement lié à l'histoire sociale et la démarche prosopographique qui consiste à enquêter sur l'origine des soldats et de leurs chefs, mais aussi sur l'organisation des différents groupes militaires à Chypre, est une source d'informations remarquable sur la société chypriote de l'époque ptolémaïque.

43. Mooren 1977, p. 36.

44. Trente-trois individus d'après le recensement opéré par I. Nicolaou. Parmi eux, 19 seraient originaires de la Grèce ou des îles (Michaelidou-Nicolaou 1969, p. 161-162).

45. S'il faut se garder d'interpréter a priori toutes les inscriptions funéraires mentionnant un ethnique étranger comme des épitaphes de mercenaires (Robert 1965, p. 181 à propos du $n^{\circ} 450$ : l'épitaphe de deux Sidoniens provenant d'Amathonte), force est de constater que les sources chypriotes sont en adéquation avec le recrutement des mercenaires de l'armée lagide : voir Launey 1987, p. 1032.

46. Hermary 1987, p. 72-75, pl. LII.

47. Michaelidou-Nicolaou 1967, $\mathrm{n}^{\circ} 13$; PРC, П.45; Voskos 1997, E22 ; KB V, n 2070.

48. Michaelidou-Nicolaou 1967, $\mathrm{n}^{\circ} 7 ; P P C, \mathrm{~N} .29$.

49. British Museum, inv. 1894.1101.158; Hermary 1987, p. 73 et n. 144.

50. Étudié en détail par Launey (1987) ; plus récemment, voir Chaniotis 2005, notamment p. 78102 ; Fischer-Bovet 2014, p. 160-197. 
En effet, en suivant, lorsque les sources épigraphiques le permettent, un individu de son point d'origine à son lieu d'établissement, nous sommes parfois en mesure de collecter des données non seulement sur l'histoire politique et militaire de la dynastie lagide, mais aussi sur la société elle-même, par le biais des rapports que cet individu entretient avec la population locale, ses institutions, ses cultes. Cette démarche nous permet d'aborder des groupes sociaux dont la voix ne se fait pas entendre dans les grandes dédicaces honorifiques mettant en scène les rois et les dignitaires rattachés à la cour lagide, ou dans certains cas l'élite locale. On peut sur ce point revenir sur une inscription bien connue de Delphes publiée en 1882 par B. Haussoulier ${ }^{51}$. Malgré son éloignement géographique, ce texte nous engage de façon poignante dans les réseaux méditerranéens de circulation des individus à l'époque hellénistique. Comme souvent à l'époque hellénistique, tout commence par une guerre et une expédition militaire. Cette inscription nous présente deux actes distincts : une lettre de la cité crétoise d'Axos envoyée à la confédération étolienne dans la première moitié $\mathrm{du} \mathrm{II}^{\mathrm{e}} \mathrm{s}$. au sujet de l'un de ses compatriotes, Ératôn et de ses descendants, ainsi que, en guise de réponse, le décret des Étoliens. La pierre présente d'abord le décret, puis la lettre. Les deux textes font référence aux péripéties d'une famille dont l'évolution est significative à la fois pour l'étude du contexte historique et celle de la société. Ératôn, un Crétois originaire de la cité d'Axos, est entrainé à Chypre au cours d'une campagne militaire. Il s'agit, selon toute vraisemblance, d'un mercenaire crétois au service des Ptolémées, ayant stationné dans l'île pendant une durée assez longue pour avoir eu le temps de s'établir sur place, se marier et donner naissance à deux fils, Épiklès et Évagoras. C'est aussi à Chypre que meurt Ératôn; dans des circonstances qui ne sont pas précisées ${ }^{52}$, Épiklès et sa mère sont faits prisonniers, le premier étant - apparemment seul - vendu comme esclave, à Amphissa. Par un heureux revers de fortune, Épiklès parvient à racheter sa liberté, et, avec elle, sa qualité de citoyen d'Axos. C'est là qu'intervient la cité crétoise qui, par l'intermédiaire de ses cosmes, confirme auprès des Étoliens l'origine et le statut d'Épiklès. À ce titre, les Étoliens décrètent que soient inscrits les droits d'Épiklès à la koinopolitéia. Ainsi s'achèvent les tourments du fils d'Ératôn. Mais l'inscription de Delphes nous apprend encore qu'Épiklès s'est finalement établi à Amphissa, où il s'est à son tour marié et a eu trois enfants : Erasiôn (?), Timônax et Mélita. Ces textes sont d'une importance capitale. Du point de vue de l'identité - question centrale dans l'étude du mercenariat hellénistique - on remarque qu'Ératôn, probablement marié à une Chypriote, nomme l'un de ses fils Évagoras, ce qui est difficilement imputable à une simple coïncidence en contexte chypriote ${ }^{53}$. Deuxièmement, ses fils, bien que nés à

51. Haussoulier 1882, p. 460-466 (Sylloge 3 , 622 ; partiellement, $I G^{2}$ IX, 1, 178). Cf. Launey 1987, I, p. 261, II, p. 1156 ; Michaelidou-Nicolaou 1969, nº 8 ; PPC, E.11, E.18, E.26, E.17, M.13, T.57; Garlan 1989, p. 87 ; Chaniotis 2005, p. 112 et surtout A. Bielman, Retour à la liberté : libération et sauvetage des prisonniers en Grèce ancienne. Recueil d'inscriptions honorant des sauveteurs et analyse critique (Études épigraphiques I), Lausanne et Paris, 1994, p. 269-270, 293, 321.

52. Il s'agit vraisemblablement d'un contexte de piraterie : Garlan 1989, p. 138 et 276.

53. On peut donc avancer deux explications : volonté de s'inscrire dans une tradition familiale (malheureusement on n'a pas trace de ces individus dans les épitaphes, et le nom de la femme 
Chypre, sont des Crétois : c'est pour la reconnaissance de sa qualité de citoyen d'Axos qu'Épiklès a dû demander l'intervention de la cité auprès des Étoliens, et c'est encore en cette qualité qu'il obtient des droits fondamentaux : droit de mariage, de participer à la vie politique et à la vie religieuse ${ }^{54}$. Outre le témoignage concret de la violence de la société dans laquelle évoluait la famille d'Ératôn et d'Épiklès, cette inscription présente un grand intérêt ${ }^{55}$ pour l'histoire de Chypre et de l'empire lagide, les institutions des cités crétoises et leurs rapports avec les cités grecques à l'époque hellénistique, et l'étude d'un groupe social directement en prise avec l'histoire des grandes monarchies hellénistiques, à savoir celui des mercenaires.

\section{Des Chypriotes actifs hors de Chypre : les réseaux externes}

Dans le cadre de cette étude générale, on peut étendre à l'île de Chypre tout entière l'interrogation formulée par Jean Pouilloux en 1973 quant au devenir des « courants » méditerranéens qui reliaient entre autres Salamine à Athènes et à Délos à l'époque hellénistique ${ }^{56}$. Le même souci anime l'enquête menée par Antoine Hermary en 1999, lorsqu'il se penche sur les quelques noms de Chypriotes retrouvés en Égypte et sur la stèle funéraire d'un Amathousien en Thessalie ${ }^{57}$. La question de la présence de Chypriotes hors de Chypre à l'époque hellénistique a ainsi souvent été posée, et elle a été ponctuellement et partiellement traitée : Inô Nicolaou donne à la fin de sa Prosopography of Ptolemaic Cyprus un index des ethniques dans lequel sont intégrés l'ethnique Kyprios et quelques autres se rapportant à l'identité des cités chypriotes. À nouveau, Inô Nicolaou, lors du colloque de 1985 à Nicosie ${ }^{58}$, s'intéresse aux Chypriotes connus à l'étranger, tandis que Jean Pouilloux, consacre plusieurs enquêtes à la relation de Chypre - notamment Salamine - avec Athènes ${ }^{59}$, Délos ${ }^{60}$ ou Delphes ${ }^{61}$.

d'Ératôn n'est pas mentionné dans les textes de Delphes) ou plutôt volonté d'inscrire la famille dans la tradition locale chypriote en faisant référence à Évagoras I, le roi de Salamine (411-374). Par ailleurs, la tradition se perpétue peut-être à la génération suivante lorsque Épiklès, fils d'Ératôn, devient lui-même le père à Amphissa d'un certain Timônax (il est certain que ce nom est répandu à Chypre, et notamment à Amathonte).

54. Haussoulier 1882, p. 465 et n. 5 .

55. Haussoulier insiste également sur l'intérêt linguistique de cette inscription dans son édition originale : Haussoulier 1882, p. 461-462.

56. Pouilloux 1973, p. 401.

57. Hermary 1999.

58. I. Nicolaou, «Cypriots in the East and West. Foreigners in Cyprus, Archaic to Roman period », dans V. Karageorghis (éd.), Acts of the International Archaeological Symposium "Cyprus between the Orient and the Occident”, Nicosia, 8-14 September 1985, Nicosie, 1986, p. 423-438.

59. J. Pouilloux, «Athènes et Salamine de Chypre », RDAC 1975, p. 111-121, pl. XV.

60. Pouilloux 1973 ; Id., «Étrangers à Kition et Kitiens à l'étranger», RDAC 1988, p. 95-99.

61. J. Pouilloux, «Chypriotes à Delphes », RDAC 1976, p. 158-167. 
En l'état actuel de nos connaissances, environ quatre-vingts individus portant un ethnique chypriote sont recensés dans les différentes sources épigraphiques de l'époque hellénistique. Ils sont dispersés entre la Grèce continentale et insulaire, l'Égypte et l'Asie Mineure. C'est dans le corpus de Délos que nous trouvons le plus grand nombre de Chypriotes. De façon générale, une dizaine d'individus portent l'ethnique Kyprios ${ }^{62}$; tous les autres sont identifiés par l'ethnique de leur cité d'origine. Salamine d'abord, puis Paphos, dominent cet ensemble, les deux grandes cités rassemblant à elles deux plus de la moitié du corpus.

Pour finir, je me pencherai sur les cas de deux Chypriotes, particulièrement bien documentés et emblématiques de la position des élites chypriotes dans les réseaux méditerranéens de l'époque hellénistique. Simalos $(P P C, \Sigma .13)$ fils de Timarchos ${ }^{63}$ est un Salaminien présent de façon relativement discrète dans le corpus des inscriptions de Chypre par la dédicace ${ }^{64} \mathrm{~d}$ 'une statue du stratège Hélénos, dans sa ville d'origine où il est alors gymnasiarque. On connaît son père ${ }^{65}$ : Timarchos, proxène et bienfaiteur d'Athènes à Salamine, est en effet honoré de façon exceptionnelle par le démos et la boulè d'Athènes dans les années 160/150. Mais c'est à Délos que l'épigraphie nous révèle l'importance du fils. Simalos s'y illustre d'abord par une dédicace ${ }^{66}$ en l'honneur de Stolos, un proche de Ptolémée Sôter II assurant les fonctions d'amiral tandis que le prince occupe en personne la fonction de stratège de Chypre. L'activité de Simalos s'inscrit donc dans le contexte troublé des rivalités dynastiques qui tendent à personnaliser les rapports entretenus par les princes lagides avec l'île de Chypre, de plus en plus envisagée comme le refuge ${ }^{67}$, sinon le quartier général des prétendants au trône d'Alexandrie.

Les deux inscriptions évoquées ci-dessus fonctionnent en miroir et sont significatives : Simalos honore à Chypre le stratège Hélénos, proche de Ptolémée X Alexandre I, avant de se rapprocher du camp adverse, en faisant dresser à Délos une statue de l'Athénien Stolos, proche de Ptolémée IX Sôter II. Le dernier document ${ }^{68}$ en notre possession dans ce dossier provient également de Délos, émis cette fois par Stolos. Il se compose d'une base de statue en l'honneur de Simalos, suivie d'une épigramme faisant l'éloge de son hospitalité et de ses attaches familiales avec l'Égypte, Rome et Athènes. La question des nombreuses relations de Simalos avec Athènes et Tarente a été traitée par Jean

62. O. Masson, « Notes d'onomastique chypriote, III », Kypriakai Spoudai 28, 1964, p. 3-12.

63. Pouilloux 1973, p. 399-413.

64. T.B. Mitford, «Helenos, Governor of Cyprus », JHS 79, 1959, p. 102, n 9 et p. 128.

65. Il est présenté par Nicolaou (PPC, T.23) comme « undoubtedly the most outstanding Cypriot, who [also] lived abroad».

66. ID 1534 .

67. G.H. Macurdy, Hellenistic queens: a Study of woman-power in Macedonia, Seleucid Syria and Ptolemaic Egypt, Chicago, 1932, p. 164 évoque : « [Cyprus], the natural refuge of a Ptolemy».

68. Voskos 1997, p. 64-67, E1. 
Pouilloux ${ }^{69}$ et témoigne de la vivacité des contacts entre Chypre, Athènes et l'Italie ${ }^{70}$. Ces preuves de la relation d'amitié réciproque entre les deux personnages montrent que le Salaminien Simalos avait pris le parti d'élargir ses réseaux et de s'affranchir de l'emprise alexandrine en allant chercher, pour lui et sa descendance, un avenir résolument tourné vers l'Occident. Il faut souligner que l'épigramme délienne destinée à Simalos porte une signature : celle d'Antisthène de Paphos ${ }^{71}$, poète fameux originaire de la capitale hellénistique de Chypre. Ce document délien présente donc un double intérêt pour l'étude de l'influence chypriote à l'étranger, dans la mesure où il éclaire le rayonnement des élites chypriotes en Méditerranée sous des aspects divers : économiques et politiques, mais aussi artistiques et culturels.

Un autre Paphien bénéficie à la fin de l'époque hellénistique d'une renommée remarquable : Onésandros $(P P C, 0.28)$ fils de Nausikratès est l'un des rares Chypriotes

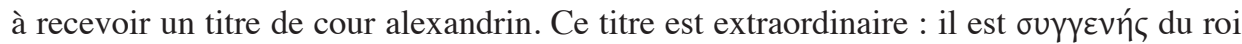
Ptolémée IX Sôter II ${ }^{72}$ et occupe les fonctions de prêtre à vie du même monarque au sein du Ptolémaion qu'il a lui-même fondé à Paphos. C'est en grammateus de la cité de Paphos qu'Onésandros débute une carrière qui atteint son sommet avec sa nomination à la tête de la Grande Bibliothèque à Alexandrie. Son ascension est, là encore, liée au même contexte politique et dynastique troublé du début du I ${ }^{\mathrm{er}} \mathrm{s}$. av. J.-C. Sa proximité avec Ptolémée IX est attestée et il ne fait nul doute que la loyauté et l'activité témoignée dans la promotion du culte du souverain expliquent en grande partie sa réussite sociale. Le passage du circuit des fonctions locales à celui de la hiérarchie de cour alexandrine est exceptionnel. Ce parcours est l'illustration de plusieurs phénomènes : le relâchement des barrières traditionnelles entre les dignitaires lagides et la population chypriote ; la décentralisation des pouvoirs dès la fin $\mathrm{du}^{\mathrm{e}}{ }^{\mathrm{e}} \mathrm{s}$., qui coïncide avec l'attribution à des princes lagides des fonctions du stratège de Chypre ; la personnalisation des rapports entretenus par les Ptolémées avec l'élite locale qui répond à l'intensification des troubles dynastiques à Alexandrie. Ainsi, c'est lorsque le trône d'Alexandrie revient à Ptolémée IX, entre 88 et 81 , qu'il peut installer son loyal Parent chypriote à la tête de l'une des plus illustres institutions du royaume lagide.

\section{Conclusions : Un autre monde?}

L'intégration de Chypre en tant qu'entité unifiée au sein d'un système politique, économique et militaire imposé par une puissance extérieure représente une réelle rupture avec la période précédente. En ce sens, la disparition des cités-royaumes et l'installation pérenne de l'appareil alexandrin peuvent être considérées comme l'entrée de Chypre dans

69. Pouilloux 1973.

70. Voir aussi PPC, $\Sigma .12, \Sigma .13$, T.20-23.

71. Voskos 1997, p. 151-177, E1.

72. Mooren 1977, p. 188-189. On peut aussi penser que ce titre correspond à une hiérarchie de cour locale et que le déplacement d'une partie de l'autorité royale à Paphos a entraîné, du point de vue des titres auliques, une adaptation et une redéfinition locales. 
un monde nouveau. Mais un monde qui garde la Méditerranée en son centre, et qui, en ce sens, n'est pas fondamentalement autre. Parce que le monde hellénistique reste un monde méditerranéen, tout ne change pas pour Chypre à la fin $\mathrm{du}_{\mathrm{IV}}^{\mathrm{e}} \mathrm{s}$. Certains phénomènes perdurent bien au-delà de la césure chronologique marquée par le début de l'installation lagide. Chypre demeure un lieu de contacts privilégiés du bassin méditerranéen, et ces contacts, hostiles ou pacifiques, ne diminuent pas au cours de la période hellénistique. Des Chypriotes sont connus à l'étranger, développant des activités au-delà de l'aire d'influence lagide, en s'appuyant sur les grands relais méditerranéens que sont, encore à l'époque hellénistique, Athènes et Délos. Les élites chypriotes manifestent toujours un sens aigu de l'intérêt politique, continuant d'adapter leur langage - leur langue d'abord, mais aussi leurs codes de communication - à celui des puissants qui les dominent.

Enfin, et c'est là le point de départ d'une nouvelle enquête épigraphique ${ }^{73}$, il y a toujours à Chypre une identité forte et des marqueurs efficaces pour la porter - comme en témoigne l'usage du syllabaire chypriote dont on ne cesse d'abaisser les limites chronologiques, au fil des découvertes et des nouveaux travaux ${ }^{74}$. C'est certainement dans l'étude de cette négociation perpétuelle entre adaptation et conservatisme que réside l'une des réponses à la question posée par les organisateurs de ce colloque ${ }^{75}$.

Aix Marseille Univ, CNRS, Minist Culture \& Com, CCJ, Aix-en-Provence, France

\section{ABRÉVIATIONS}

ID : Inscriptions de Délos, Paris, 1926-1972.

IG : Inscriptions Graecae, Berlin, 1815-.

I. Kourion : T.B. Mitford, The Inscriptions of Kourion (Memoirs of the American Philosophical Society 83), Philadelphie, 1971.

KB V : M. Yon (dir.), Kition Bamboula V. Kition dans les textes, Paris, 2004.

LBW : Ph. Le Bas, W.H. Waddington, Voyage archéologique en Grèce et en Asie Mineure, vol. III, Paris, 1870.
$P P C$ : I. Michaelidou-Nicolaou, Prosopography of Ptolemaic Cyprus (SIMA 44), Göteborg, 1976.

Sal. XIII : J. Pouilloux, P. Roesch, J. MarcilletJaubert, Salamine de Chypre XIII, Testimonia Salaminia 2, Paris, 1987.

Sylloge $e^{3}$ W.Dittenberger, Sylloge Inscriptionum Graecarum, Leipzig, 1915-1924.

73. C'est l'un des axes de mon travail doctoral.

74. Lejeune 2014.

75. C'est en leur adressant mes profonds remerciements pour l'invitation à participer à cet hommage à Antoine Hermary que je souhaite clore ces quelques réflexions sur Chypre à l'époque hellénistique. Ma reconnaissance va également à mon professeur, pour l'attention et la bienveillance avec lesquelles il encadre mes travaux de thèse. 


\section{BIBLIOGRAPHIE}

Anastassiades (A.), 1998, « 'Apolvóns $\Phi 1 \lambda \alpha \delta \varepsilon \dot{\lambda} \varphi \mathrm{v}$ : Aspects of a specific cult in Cyprus », RDAC, p. 129-140, pl. XIV.

Bagnall (R.S.), 1976, The Administration of the Ptolemaic Possessions outside Egypt, Leyde.

Balandier (C.) éd., 2016, Nea Paphos : fondation et développement urbanistique d'une ville chypriote de l'antiquité à nos jours. Études archéologiques, historiques et patrimoniales, Bordeaux.

CAYla (J.-B.), 2003, Les inscriptions de Paphos. Corpus des inscriptions alphabétiques de Palaipaphos, de Néa Paphos et de la chôra paphienne, thèse (inédite), université Paris IV-Sorbonne.

CaYla (J.-B.), 2006, « Liens commerciaux et alliances matrimoniales entre Chypriotes et négociants romains», dans S. Fourrier, G. Grivaud éd., Identités croisées en un milieu méditerranéen : le cas de Chypre (Antiquité - Moyen Âge), Rouen, p. 187-205.

Chaniotis (A.), 2005, War in the Hellenistic world: A social and cultural history, Oxford.

Fischer-Bovet (Chr.), 2014, Army and society in Ptolemaic Egypt, Cambridge.

Garlan (Y.), 1989, Guerre et économie en Grèce ancienne, Paris.

Haussoulier (B.), 1882, «Inscriptions de Delphes », BCH 6, p. 445-466.

Hermary (A.), 1987, « Statuettes, sarcophages et stèles décorées », dans La nécropole d'Amathonte, tombes 113-367 (ÉtChyp IX), Nicosie, p. 53-75, pl. XLII-LII.

Hermary (A.), 1999, « Des Chypriotes loin de leur île (époque hellénistique) », CCEC 29, p. 45-59.

Launey (M.), 1987 [1949-1950], Recherches sur les armées hellénistiques, $2^{2}$ éd., Paris.

LeJeune (S.), 2014, « Le sanctuaire de Kafizin : nouvelles perspectives », $B C H \quad 138$, p. 245-327.
Mehl (A.), 2016, « Nea Paphos et l'administration ptolémaïque », dans Balandier 2016, p. 249-260.

Michaelidou-Nicolaou (I.), 1967, « The Ethnics in Hellenistic Cyprus I, The Epitaphs », Kypriakai Spoudai 31, p. 15-36.

Michaelidou-Nicolaou (I.), 1968, « The Ethnics in Hellenistic Cyprus II, The Honorific Inscriptions », Kypriakai Spoudai 32, p. 23-42.

Michaelidou-Nicolaou (I.), 1969, « The Ethnics in Hellenistic Cyprus III, The dedicatory inscriptions and other documents », Kypriakai Spoudai 33, p. 155-165.

Mitford (T.B.), 1953, « The Character of the Ptolemaic Rule in Cyprus », Aegyptus 33, p. 80-90.

Mitford (T.B.), 1961, « The Hellenistic inscriptions of Old Paphos », ABSA 56, p. 1-41.

MŁynarczyk (J.), 1990, Nea Paphos in the Hellenistic Period, Varsovie.

MoORen (L.), 1977, La hiérarchie de cour ptolémaïque : contribution à l'étude des institutions et des classes dirigeantes à l'époque hellénistique (Studia Hellenistica 23), Louvain.

Papantoniou (G.), 2012, Religion and social transformations in Cyprus: From the Cypriot Basileis to the Hellenistic Strategos, Oxford.

Pouilloux (J.), 1973, « Salaminiens de Chypre à Délos », dans Études déliennes (BCH Suppl. 1), p. 399-413.

Robert (J. et L.), 1965, « Bulletin épigraphique », REG, p. 70-204.

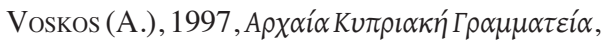
vol. 2, Nicosie.

WILL (Éd.), 2003, Histoire politique du monde hellénistique de 323 à 30 av. J.-C., $2^{\mathrm{e}}$ éd., Paris. 